\title{
¿Qué nos ha legado el pasado? ¿Qué nos queda por hacer?
}

Shannon Botelho*

\section{Resumen}

En este artículo se reflexiona sobre la escena artística y cultural de los países sudamericanos y los conflictos políticos que rodean este lugar de pensamiento. A través del uso de los conceptos de colonización, posmodernidad y descolonización se presentan formas de entender las relaciones artísticas y culturales contemporáneas, así como perspectivas para repensar los modos de conexión internacional del conocimiento. Frente a los avances en las políticas de exclusión y violencia en Brasil y Colombia se establece una plataforma de pensamiento que analiza, por un lado, el pasado y las marcas de los procesos de dominación y control intelectual y social, por el otro, el presente de las relaciones culturales entre países y formas, posibles puntos de conexión, así como puntos de vista críticos para un futuro deseado y diferente de la realidad actual.

Palabras clave: arte, cultura, crítica de arte, descolonización, posmodernidad

\section{Abstract}

This paper reflects on the artistic and cultural scene of the South American countries and the political conflicts surrounding this place of thought. Through the use of the concepts of colonization, postmodernity and decolonization, ways of understanding contemporary artistic and cultural relations are presented, as well as perspectives to rethink the modes of international connection of knowledge. In the face of advances in exclusion and violence policies in Brazil and Colombia, a thinking platform is established that analyzes, on the one hand, the past and the marks of the processes of domination and intellectual and social control, on the other, the present of cultural relations between countries and forms, possible points of connection, as well as critical points of view for a desired future and different from the current reality.

Keywords: art, art criticism, culture, decolonization, postmodernism

\footnotetext{
* Maestro en Historia y Crítica del Arte del Programa de Postgrado en Artes Visuales e Historiador del Arte de la Universidad Federal de Río de Janeiro y licenciado en Artes Visuales del Centro Universitario Metodista Bennett. Investiga el arte brasileño y sus instituciones en el siglo xx, con énfasis en la década del cincuenta. Curador, crítico de arte y profesor del Departamento de Artes Visuales del Colegio Pedro II y curador invitado en el Memorial Municipal Getúlio Vargas (RJ). Contacto: shannonbotelho@cp2.g12.br
} 
Este texto propone una doble reflexión para los ciudadanos de países peroféricos: la primera, se refiere a un rescate histórico necesario al considerar cuánto se ha avanzado en prácticas culturales; la segunda, una proposición que busca abrir una red de diálogos que nos ayude a repensar prácticas que se disuelven en la cotidianidad y resaltar aquello que aún no se ha realizado.

El escenario actual de nuestros países impone el rigor de una toma de posición analítica con foco en la reflexión, sin festejos o conmociones. La difícil tarea de producir conocimiento en el campo híbrido de las artes visuales nos cabe como artistas, críticos, curadores o historiadores del arte. En palabras del dramaturgo e intelectual brasileño Ariano Suassuna, "no soy ni optimista ni pesimista. Los optimistas son ingenuos y los pesimistas amargos. Soy un realista esperanzado" (9 de noviembre del 2012, párr. 13). A partir de esta lógica, sin perder la dimensión de la dura realidad que enfrentamos y enfrentaremos de aquí en adelante, se busca trabajar con esperanza.

En este sentido, se plantea una breve reflexión sobre las teorías y medios expresivos, desde la mitad del siglo $\mathrm{xx}$, para examinar principalmente las nociones de posmodernidad y de colonización, a fin de comprender lo que nos ha legado este pasado reciente y compartir posibilidades de continuidad para las luchas en curso. Se hace necesario, en este punto, reconocer algunos agentes que actuaron en diferentes frentes en América del Sur en defensa de nuestra producción artística, cultural e intelectual.

Las vanguardias artísticas operaron cambios paradigmáticos en el seno de la cultura social europea y norteamericana en la primera mitad del siglo xx; así, se configura como la punta de un proceso histórico que presentó los límites de su actuación, capacidad de construcción y deconstrucción. Des- de el final de los años cuarenta, las estructuras del sistema capitalista alteraban el blanco de sus acciones, configurando una nueva geopolítica mundial. De estos movimientos nacen proposiciones descolonizantes originadas en estos lugares al margen.

Una nueva palabra ingresó en el vocabulario de los debates intelectuales: posmodernidad/postmodernismo._A mediados de los ochentas, una palabra prima hermana de aquella se hizo presente: poscolonialidad/poscolonialismo. "Descolonización" pasó al segundo plano, "superada" por el pos de la modernidad. Juntas, posmodernidad y poscolonialidad, tuvieron un impacto notable en Estados Unidos principalmente, luego en Inglaterra y muy recientemente en otros países de Europa. (Gómez y Mignolo, 2012, p. 7)

En la década del sesenta, específicamente en Brasil, algunos críticos de arte contribuyeron y discutieron con movimientos de contraposición a los intentos de dominación de los medios artísticos. De esta manera, es imprescindible destacar la importancia del experimentalismo para el arte brasileño. En Experimentar lo experimental, Hélio Oiticica (2015) indica que, en el campo artístico, el espacio tradicional de contemplación cede progresivamente a la experiencia, en un modelo ampliado y continuo de crítica de la realidad cotidiana.

El carácter experimental del arte brasileño contribuía con el discurso ya conocido sobre una aproximación entre arte y vida. Por un lado, esta aproximación expandía los límites del dominio creativo e incluía en el ámbito de la producción artística a la poesía, la arquitectura, la vestimenta y la música, no solo como parte de una actitud experimental, sino como conciencia 
interdisciplinaria. De otro lado, hacía consciente de sus responsabilidades políticas al público y al sistema artístico, con la motivación de sus agentes a comprometerse en pro de acciones contestatarias y de los intentos de subversión a las fuerzas de opresión. Sobre este aspecto, el crítico de arte Mario Pedrosa aclara que:

La extrema complejidad de la civilización moderna no permite a ninguna actividad de orden científico, cultural o estético desenrollarse en el aislamiento que impone una actividad globalizante en todos los sentidos [...] además de su tendencia globalizante, la época es típica de transformaciones técnicas y sociales que se suceden día a día, reacondicionando incesantemente a la humanidad en todos los campos. (2015, pp. 433-434)

La inquietud con las nuevas formas de vivir el mundo también puede ser percibida en otros países de América del Sur. En las décadas del sesenta y setenta, marcadas por la opresión y la privación de la libertad, se imponía lentamente al sistema artístico una heroica tarea de resistir o liberar, como expone Federico Morais. Cuando las estructuras nacionalistas de los Estados modernos se flexibilizan, Brasil empieza a conocerse con el resto de América Latina. Así, se desarrolla cierta proximidad, sobre todo como estrategia de defensa frente a las presiones internacionales que vislumbraban nuevos mercados.

Juntos tenemos que elaborar las teorías capaces de hacer avanzar nuestra producción artística, juntos tenemos que promover dentro y fuera del continente nuestros artistas y las tendencias estéticas aquí nacidas. Sólo así podremos evitar el bloqueo de las multinacionales del mercado de arte y la colonización impuesta por las grandes muestras internacionales. Resistir y liberar. Pero resistir con nuevos lenguajes, o mejor, con lo nuevo. Somos la diferencia que necesitan para activar su propio proceso creador. Somos los bárbaros de una época futura. (Morais, 1979, p. 13)

Entre las propuestas que se desarrollan en los demás países sudamericanos, la tesis de Marta Traba anuncia una consonancia inédita entre los comentaristas y críticos de los procesos artísticos. En su libro Dos décadas vulnerables en las artes plásticas latinoamericanas 1950-1970, alerta que crecía a gran escala la presión políticoeconómica emanada de las naciones hegemónicas -principalmente de los Estados Unidos- y que, en las producciones locales, provocadas por estas fuerzas, se adopta una postura combativa como modo de resistencia. Así, dispone los países, de lo que define como América Latina, en áreas abiertas y cerradas. Las primeras estarían compuestas por el contrapunto del enclausuramiento, mientras que las segundas por los espacios donde la tradición y la clausura cultural predominan.

Así como el área cerrada es una categoría, donde predominan las condiciones endogámicas, la clausura, el peso de la tradición, la fuerza de un ambiente, el imperio de la raza india, de la negra, y de sus correspondientes mezclas con la raza blanca, el área abierta está marcada por su progreso, su afán civilizador, su capacidad de absorber y recibir al extranjero, su amplitud de intereses y su tendencia a la glorificación de las capitales. (Traba, 1978, p. 41)

La posición de Traba complementa el apunte de Morais en su reflexión en el sentido de la resistencia al elemento externo que asume el 
lugar del colonizador. Pero, ¿qué son estos elementos sino mecanismos intrínsecos del mercado internacional que pertenecen a los antiguos colonizadores? La pregunta que aún cabe hacer sobre este aspecto de la novedad poscolonial es: ¿quién asumirá el lugar del colonizador en los procesos mercadológicos, culturales e intelectuales?

En el intento de responder propiamente a esta cuestión que involucra el papel -ahora vago- del colonizador, Pedro Gómez y Walter Mignolo reflexionan sobre las razones de la descolonización y de la tarea común a los países recién liberados. La decolonialidad, como proposición, resultaría en la conciencia de que existe una herida colonial que habría marcado profundamente los modos de pensar y actuar, de producir y realizar, de organización política y de conocimiento. De esta manera, en el campo de las artes visuales la propuesta decolonial vislumbraría la tarea de descolonizar el propio arte y rehacer los caminos que servían de conducto a los colonizadores para dar formato a los procesos artísticos a fin de colonizar las subjetividades.

La garantía de salud, educación y alimentación necesita la afirmación de las subjetividades difamadas y devaluadas por 500 años de instauración de un orden económico y político apoyado por valores culturales y estéticos, por un ideal de humanismo y de ser humano que se auto construyó para beneficio de aquellos que le construyeron. El arte y la estética fueron instrumentos de colonización de subjetividades y hoy la descolonización de la estética para liberar la aesthesis es un aspecto fundamental del proceso de decolonialidad. (Gómez y Mignolo, 2012, p. 13)
En resumen, los países subdesarrollados optaron por la vía de la descolonización; este proceso se debió, sobre todo, a la necesidad de descubrir un espacio de libertad. En América del Sur, como se ha dicho, intelectuales y artistas procuraron esbozar proyectos que respondieran a todas las provocaciones resultantes de este proceso. En todos (de Pedrosa a Traba, de Morais a Gómez y Mignolo) resaltan los intentos de contribuir con realidades más efectivas y pertinentes al nuevo ordenamiento de la geopolítica mundial. En este sentido, se evidencia que "la tarea fundamental de los proyectos decoloniales es invertir el proceso y poner la vida de los seres humanos y del planeta, en primer lugar, y las instituciones al servicio de ella" (Gómez y Mignolo, 2012, p. 15) y que, por lo tanto, los aspectos ligados a las culturas locales que fundamentan el pensamiento posmoderno definen modos de existir en la realidad.

El mundo que conocemos es desigual, imperialista, marcado por la violencia, por la adhesión voluntaria a la ignorancia y a la facilidad del consumo, y por el desprecio a las culturas. Vuelve la pregunta inicial: ¿qué nos queda por hacer? Tal vez sea esta la pregunta más difícil que podríamos formular. De hecho, es preciso cuestionarnos por la posibilidad que tenemos hoy para contribuir a la sociedad a la que pertenecemos, sin que nuestras acciones se pierdan en el hermetismo de las producciones contemporáneas que atienden más al mercado de arte $\mathrm{y}$ a las academias que a las personas a las que se destina su existencia. ¿En qué puntos podemos avanzar en formulaciones poéticas para reencantar el mundo? Estas cuestiones no se cierran aquí, podemos aprovechar los desdoblamientos de este encuentro para responderlas. Sin embargo, es posible cerrar con tres apuntes. 
El primero, se refiere a la necesidad de articulación entre los jóvenes artistas, críticos y curadores. Es necesario que los compromisos mercadológicos que se imponen a estas relaciones no sean mayores y más anchos que las relaciones afectivas que se pueden establecer. Como en la década del cincuenta, cuando críticos y artistas se unían en sus talleres para pensar sus producciones y escribir textos manifiestos, es preciso, aún hoy, que estemos juntos, atentos, unidos contra la represión, contra la violencia física $\mathrm{y}$, sobre todo, contra las violencias simbólicas que callan nuestras voces, matan nuestros sueños y amenazan nuestro futuro.

El segundo, la necesidad de construir puentes entre islas aisladas. Así como hacemos hoy aquí, establecer conexiones intelectuales y de trabajo efectivo en el campo de las artes visuales y de la cultura. Hemos sido y seguimos siendo un continente que se desconoce; no solo se desconoce su desigualdad, hambre y pobreza, sino también su poesía, arte y pensamiento. Así como hicieron Marta Traba, Federico Morais y tantos otros, en los años setenta -época en que pensaban la situación de los artistas en sus países-, es necesario que trabajemos juntos, que conozcamos nuestras debilidades para que trabajemos en ellas.

Por último, en tercer lugar, hay que hacer la vida poética otra vez. La producción artística contemporánea se ha alejado de la poesía por considerar que esta se relaciona directamente con la belleza. Necesitamos afirmar que no, la poesía y la belleza están en lugares distintos (aunque pueden coexistir). Pero la poesía es la única que puede vigilar contra la inhumanidad, la rudeza de las costumbres y la mediocridad de las acciones violentas de lo cotidiano. Con poco, gestos o acciones, es posible transfigurar la realidad. No nos queda el objeto artístico, pues fue desmaterializado por la vanguardia; no nos queda la belleza, fue superada en la modernidad, y no nos queda la técnica, no se aplica más en el mundo posmoderno. Nos queda nuestra propia existencia. Entonces, lo que nos queda hacer es existir, aunque no nos quieran, resistir a la violencia y el dolor, reexistir con poesía e inteligencia. 


\section{Referencias}

Gómez, P. y Mignolo, W. (2012). Estéticas decoloniales [recurso en línea]. Bogotá: Universidad Distrital Francisco José de Caldas. Recuperado de https:/ / bit.ly/3aG7Jga

Morais, F. (1979). Artes plásticas na América Latina: do transe ao transitório. Rio de Janeiro: Civilização Brasileira.

Oiticica, H. (2015). Experimentar lo experimental. Cuenca, Ecuador: Fundación Municipal Bienal de Cuenca.

Pedrosa, M. (2015). Arte. Ensaios. San Pablo: Cosac Naify.

Suassuna, A. (9 de noviembre del 2012). 'Tenho certeza que Lula Não sabia de nada', afirma Suassuna sobre o mensalão. Entrevista con E. de Lucena. Folha de S. Paulo. Recuperado de https:/ / bit.ly/2Gr70Bw

Traba, M. (1978). Duas décadas vulneráveis nas artes plásticas latino-americanas, 1950/1970. Rio de Janeiro: Paz e Terra. 